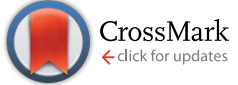

Cite this: RSC Adv., 2017, 7, 6856

Received 7th December 2016 Accepted 12th January 2017

DOI: 10.1039/c6ra27901e

www.rsc.org/advances

\title{
Amorphous molybdenum sulfide on graphene- carbon nanotube hybrids as supercapacitor electrode materials
}

\begin{abstract}
Kien-Cuong Pham, ${ }^{\text {ab }}$ David S. McPhail, ${ }^{c}$ Andrew T. S. Wee ${ }^{\text {ad }}$ and Daniel H. C. Chua*e
Herein, we report the application of amorphous molybdenum sulfide $\left(\operatorname{MoS}_{x}, x \approx 3\right)$ as the main active material for supercapacitor electrodes. MoS $_{x}$ was deposited at room temperature onto a high specific surface area electrode made of graphene-carbon nanotube hybrids directly grown on carbon paper (GCNT/CP), using an electrochemical deposition method. The $\operatorname{MoS}_{x} /$ GCNT/CP electrode showed high specific capacitance. A gravimetric specific capacitance of $414 \mathrm{~F} \mathrm{~g}^{-1}$ was demonstrated at a constant discharge rate of $0.67 \mathrm{~A} \mathrm{~g}^{-1}$. The deposition of $\mathrm{MoS}_{x}$ onto a conductive, high surface area support played a crucial role for a high specific capacitance. An up to 4.5-fold enhancement in specific capacitance was demonstrated when MoS $_{x}$ was deposited on GCNT/CP as compared to MoS deposited on a simple carbon paper support. The $\mathrm{MoS}_{x} / \mathrm{GCNT/CP}$ electrode is suggested to be a novel candidate for supercapacitor applications.
\end{abstract}

\section{Introduction}

Renewable energy solutions are being very actively sought, due to energy security and environmental concerns. Among these, supercapacitors have recently attracted much research attention for their high power density, and quick charge/discharge capability. ${ }^{1-3}$ Supercapacitors frequently make use of high surface area carbon-based materials, such as activated carbon, ${ }^{4}$ graphene ${ }^{2,5}$ and carbon nanotubes, ${ }^{6}{ }^{6}$ as electrode materials. However, the reported specific capacitances of carbon-based electrodes have been relatively low. Recently, other electrode materials have been actively investigated for supercapacitor applications, for example, metal oxides, ${ }^{7,8}$ metal hydroxides, ${ }^{9,10}$ metal sulfides, ${ }^{11,12}$ to name a few. Transitional metal chalcogenides such as $\mathrm{WS}_{2}, \mathrm{MoS}_{2}, \mathrm{Ni}_{3} \mathrm{~S}_{4}, \mathrm{MoSe}_{2}$, and CuS have recently emerged as promising supercapacitor electrode materials., ${ }^{3,11-23}$ One notable example in this family of materials is molybdenum disulfide $\left(\mathrm{MoS}_{2}\right) \cdot \mathrm{MoS}_{2}$ is used as a supercapacitor electrode material due to its higher ionic conductivity than metal oxides

${ }^{a}$ NUS Graduate School for Integrative Sciences and Engineering (NGS), National University of Singapore, 28 Medical Drive, Singapore 117456, Singapore. E-mail: phamkiencuong@u.nus.edu

${ }^{b}$ Department of Materials, Imperial College London, Exhibition Road, London SW7 2AZ, $U K$

'Department of Chemistry and Biochemistry, University of Texas at Dallas, TX 750803021, USA. E-mail: dsm160330@utdallas.edu

${ }^{d}$ Department of Physics, National University of Singapore, 2 Science Drive 3, Singapore 117542, Singapore. E-mail: phyweets@nus.edu.sg

${ }^{e}$ Department of Materials Science and Engineering, National University of Singapore, 9 Engineering Drive 1, Singapore 117576, Singapore.E-mail: msechcd@nus.edu.sg; Fax: +65-6776-3604; Tel: +65-6516-8933 and its higher specific capacitance than graphite., ${ }^{3,17,18}$ While much research attention has been paid to crystalline $\mathrm{MoS}_{2},{ }^{3,12,15-18}$ little attention has been paid to its amorphous form, except for one recent study reporting the use of amorphous $\mathrm{MoS}_{2}$ as an encapsulating material for $\mathrm{Ni}_{3} \mathrm{~S}_{4} \cdot{ }^{19}$

Amorphous molybdenum sulfide $\left(\operatorname{MoS}_{x}\right)$ possesses many advantageous merits that can warrant further attention. In supercapacitors, charge storage in crystalline $\mathrm{MoS}_{2}$ can occur via the double layer charging on the external surface of the material or via the diffusion of ions into the inter-layer space of $\operatorname{MoS}_{2} \cdot{ }^{14,17,18}$ The diffusion of ions into the inter-layer space occurs through the open edge sites of $\mathrm{MoS}_{2}$, due to the anisotropic layered structure of crystalline $\mathrm{MoS}_{2}$. Naturally occurring crystalline $\mathrm{MoS}_{2}$ has a layered crystal structure similar to graphite. To enhance this diffusion process, Soon and Loh strategically oriented the open edge planes of $\mathrm{MoS}_{2}$ to maximize the availability of these "entry" sites. ${ }^{14}$ In contrast, the amorphous form of materials generally has more open and isotropic atomic structures. ${ }^{24}$ The diffusion of ions in $\operatorname{MoS}_{x}$ is therefore not limited by the availability of edge sites. The diffusion of ions may happen throughout the entire material surface. The diffusion coefficient of ions (e.g. lithium) in $\mathrm{MoS}_{x}$ was found to be several orders of magnitude higher than that in crystalline $\mathrm{MoS}_{2}{ }^{24,25}$ The electrical conductivity of $\mathrm{MoS}_{x}$ was also found to be two orders of magnitude higher than that of crystalline $\operatorname{MoS}_{2} \cdot{ }^{24}$ The higher electronic conductivity of $\operatorname{MoS}_{x}$ is beneficial for a better supercapacitive performance. Furthermore, in layered materials such as graphene and $\mathrm{MoS}_{2}$, it was suggested that edge sites and defect sites in the material structures were more electro-active than the basal plane atoms and can enhance electrode capacitance. ${ }^{26} \operatorname{MoS}_{x}$ intrinsically contains abundance 
of defect sites. The contribution of defect sites in $\operatorname{MoS}_{x}$ to the total capacitance is non-trivial and deserves further attention. As an additional advantage, $\operatorname{MoS}_{x}$ syntheses can be routinely done at ambient temperature using wet chemical syntheses ${ }^{27,28}$ or electrodeposition methods, ${ }^{29,30}$ in contrast to the higher temperature syntheses of crystalline $\mathrm{MoS}_{2}$. Herein, we investigate molybdenum sulfide in its amorphous form as the main active supercapacitor material.

Although molybdenum sulfide materials potentially have higher specific capacitance than graphite, they are generally less electronically conductive than graphitic materials. ${ }^{3}$ The poor electronic conductivity of molybdenum sulfide could result in low material utilization and poor high-discharge-rate performance. Depositing $\operatorname{MoS}_{x}$ on a high surface area conductive support can be an effective approach to enhance the electron transport between the active material and the current collector, and therefore maximize the electrode capacitance.

In our previous studies, we reported a hierarchical electrode structure of graphene-carbon nanotube hybrids grown directly on carbon paper (GCNT/CP). ${ }^{31-35}$ In this structure, CNTs were firstly grown on Toray carbon paper, and free-standing graphene was then grown directly on CNT scaffolds. The electrode had an integrated, binder-free, high specific surface area, and conductive structure. $\operatorname{MoS}_{x}$ was also successfully deposited on the GCNT/CP support for the Hydrogen Evolution Reaction (HER) application in our previous study, reporting one of the most active molybdenum sulfide-based HER catalysts to date. ${ }^{33}$ Herein, the supercapacitive properties of $\mathrm{MoS}_{x}$ and specific capacitance enhancement effects of the $\mathrm{MoS}_{x} / \mathrm{GCNT} / \mathrm{CP}$ electrode will be reported.

\section{Experimental}

\subsection{Growth of the GCNT hybrids}

The GCNT hybrids were grown on carbon paper using the Chemical Vapor Deposition (CVD) methods in a two-step procedure. The details of growth procedure and the hybrid structures have been previously reported elsewhere. ${ }^{31,32,34}$ To summarize, CNTs were firstly grown on a carbon paper substrate using the thermal CVD technique. Subsequently, freestanding graphene was grown on the CNT scaffolds using the catalyst-free radio frequency plasma enhanced CVD.

\subsection{Deposition of $\operatorname{MoS}_{x}$ on the GCNT/CP support}

$\operatorname{MoS}_{x}$ was deposited onto the GCNT/CP electrode using an electrodeposition method as previously reported..$^{33}$ To summarize, $\operatorname{MoS}_{x}$ was firstly electrodeposited on the GCNT/CP support from a $20 \mathrm{mM}$ aqueous solution of ammonium tetrathiomolybdate $\left(\left(\mathrm{NH}_{4}\right)_{2} \mathrm{MoS}_{4} 99.97 \%\right.$, Sigma-Aldrich), performed in a three-electrode electrochemical cell. The deposition was carried out by holding the GCNT/CP working electrode at $0.7 \mathrm{~V}$ vs. Standard Hydrogen Electrode (SHE) potential. Subsequently, the $\mathrm{MoS}_{x}$-deposited GCNT/CP electrode underwent 10 reductive potential sweeps from 0.2 to $-0.5 \mathrm{~V} v$ s. SHE in a $0.5 \mathrm{M} \mathrm{H}_{2} \mathrm{SO}_{4}$ electrolyte solution. In a typical experiment, the final $\mathrm{MoS}_{x}$ loading was approximately $3 \mathrm{mg} \mathrm{cm}^{-2}$. In all subsequent discussions, $\operatorname{MoS}_{x} / \mathrm{GCNT} / \mathrm{CP}$ refers to this final condition of the electrode. For comparisons, $\operatorname{MoS}_{x}$ was also deposited onto a carbon paper substrate $\left(\mathrm{MoS}_{x} /\right.$ carbon paper) using the same aforementioned procedure and $\operatorname{MoS}_{x}$ loading of $3 \mathrm{mg} \mathrm{cm}{ }^{-2}$.

\subsection{Material characterization}

The morphological characterization of the GCNT/CP and $\mathrm{MoS}_{x} /$ GCNT/CP electrodes was performed with Field Emission Scanning Electron Microscopy (FE-SEM, JEOL JSM-6700F). The chemical analyses were characterized with X-ray Photoelectron Spectroscopy (XPS) (Omicron 7-channeltron analyzer, $\mathrm{Al} \mathrm{K}_{\alpha} \mathrm{X}$ ray source of $1486.7 \mathrm{eV}$ photon energy, Omicron X-ray twin anode source). Constant analyzer energy (CAE) mode of $20 \mathrm{eV}$ pass energy was used for high resolution scans. The amorphous nature of $\operatorname{MoS}_{x}$ was assessed by Raman spectroscopy (Renishaw inVia Raman microscope, $532 \mathrm{~nm}$ laser) and X-ray diffraction (XRD) (Philips X'Pert, $\mathrm{Cu} \mathrm{K}_{\alpha}(\lambda=1.54 \AA$ ) source).

\subsection{Electrochemical measurements}

Supercapacitive properties of the $\mathrm{MoS}_{x} / \mathrm{GCNT} / \mathrm{CP}$ electrode were measured in a three-electrode cell configuration. The $\operatorname{MoS}_{x}$ l GCNT/CP electrode was used as working electrode. A platinum rod, a $\mathrm{Ag} / \mathrm{AgCl}(3 \mathrm{M} \mathrm{KCl})$ electrode and a $1 \mathrm{M} \mathrm{Na}_{2} \mathrm{SO}_{4}$ solution were used as counter electrode, reference electrode and electrolyte, respectively. The neutral $1 \mathrm{M} \mathrm{Na}_{2} \mathrm{SO}_{4}$ electrolyte was used instead of acidic $\mathrm{H}_{2} \mathrm{SO}_{4}$ electrolyte to avoid the hydrogen evolution process. The $\mathrm{MoS}_{x} / \mathrm{GCNT} / \mathrm{CP}$ electrode has been shown to be highly active towards HER in acidic electrolyte in our previous study. ${ }^{33}$ All electrodepositions and electrochemical measurements were carried out with an Autolab PGSTAT302N potentiostat/galvanostat (Metrohm), fitted with a FRA2.V10 frequency response analyzer and a SCANGEN analog scan generator. All potentials in the following contexts were reported against SHE. Cyclic voltammetry and galvanostatic charge/ discharge experiments were carried out in a potential window of between -0.5 and $0.3 \mathrm{~V} v$ s. SHE. Electrochemical Impedance Spectroscopy (EIS) was measured at $-0.1 \mathrm{~V}$ vs. SHE in a frequency range of between $10 \mathrm{kHz}$ and $0.01 \mathrm{~Hz}$ with an AC perturbation voltage of $5 \mathrm{mV}$. Stability testing was performed using 500 galvanostatic charge/discharge cycles at a constant charge/discharge rate of $5 \mathrm{~A} \mathrm{~g}^{-1}$.

\section{Results and discussion}

\subsection{Physical properties of the GCNT hybrids and the $\operatorname{MoS}_{x} /$ GCNT/CP electrode}

The morphological study with FE-SEM of the GCNT/CP and $\mathrm{MoS}_{x} / \mathrm{GCNT} / \mathrm{CP}$ electrodes is provided in Fig. 1. Fig. 1a shows an SEM micrograph of the GCNT/CP support. The porous and high surface area structure of the GCNT/CP support was clearly observed. The GCNT hybrids possessed a unique morphology with high density of free-standing graphene deposited on the fibrous CNT scaffolds. The CNT scaffolds have two main roles in the GCNT/CP electrode structure. Firstly, as opposed to regular two-dimensional graphene sheets which have tendency to restack and block the mass transport pathways, CNTs provide 


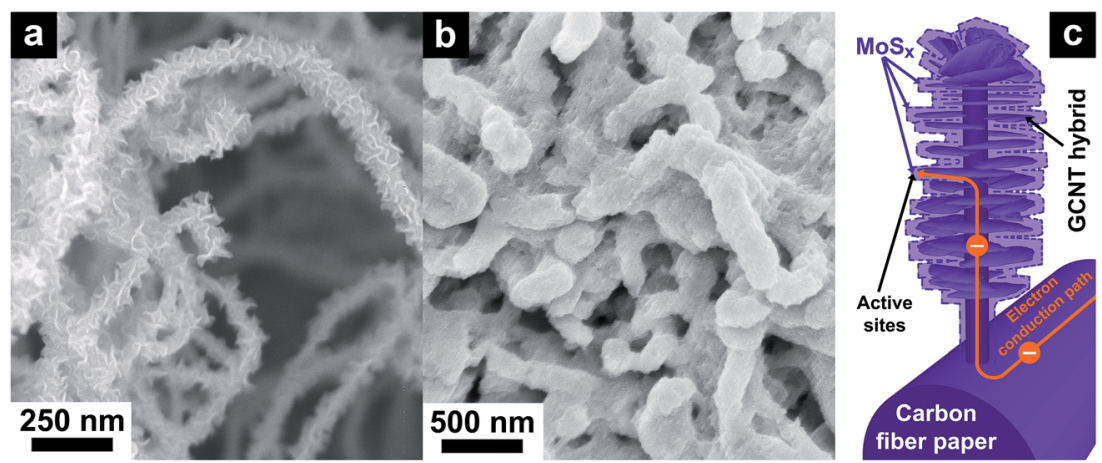

Fig. 1 Morphological characterization with FE-SEM of (a) the GCNT/CP support, and (b) the MoS $/$ GCNT/CP electrode. (c) Schematic illustration of the $\operatorname{MOS}_{x} /$ GCNT/CP electrode structure.

a porous overall electrode structure. Such a porous electrode structure could better facilitate the permeation of electrolyte solution towards the active material of $\operatorname{MoS}_{x}$. Secondly, the direct growth of CNTs on carbon paper can provide better mechanical integrity and more direct electrical conducting paths. The porous and high surface area structure of the GCNT hybrids could facilitate the deposition of $\operatorname{MoS}_{x}$ with good material dispersion, good electron and electrolytic ion transport. Further characterization of the GCNT/CP support with TEM, Raman spectroscopy, and XPS can be found in our previous study. ${ }^{31}$ Fig. $1 \mathrm{~b}$ shows an SEM micrograph of the $\operatorname{MoS}_{x} /$ GCNT/CP electrode. $\operatorname{MoS}_{x}$ was successfully deposited on the high specific surface area GCNT/CP support. $\mathrm{MoS}_{x}$ was found to coat and disperse well on the conductive support, which is highly beneficial for the enhanced electron transport towards the active material $\operatorname{MoS}_{x}$. A schematic illustration of the $\operatorname{MoS}_{x} /$ GCNT/CP electrode structure is shown in Fig. 1c.

The chemical state of $\operatorname{MoS}_{x}$ was assessed with XPS. Fig. 2a shows the high resolution XPS scan in the $\mathrm{S} 2 \mathrm{p}$ region of spectrum. The spectrum was deconvoluted into two doublets. The $\mathrm{S}^{(\mathrm{A})} 2 \mathrm{p}$ doublet at Binding Energy (BE) of 162.9-164.1 eV was assigned to apical $\mathrm{S}^{2-}$ or bridging $\mathrm{S}_{2}{ }^{2-} .{ }^{36}$ The $\mathrm{S}^{(\mathrm{B})} 2 \mathrm{p}$ doublet at $\mathrm{BE}$ of $161.4-162.6 \mathrm{eV}$ was assigned to basal plane $\mathrm{S}^{2-}$ or terminal $\mathrm{S}_{2}{ }^{2-} \cdot{ }^{36}$ Fig. $2 \mathrm{~b}$ shows the high resolution XPS scan in the Mo 3d-S 2s region. The spectrum was deconvoluted into four peak sets, including two S 2s peaks at BE of 227.3 and $226.1 \mathrm{eV}$, corresponding to the respective $\mathrm{S}^{(\mathrm{A})}$ and $\mathrm{S}^{(\mathrm{B})}$ chemical states, a $\mathrm{Mo}^{(\mathrm{A})} 3 \mathrm{~d}$ doublet at $\mathrm{BE}$ of $231.85-235.0 \mathrm{eV}$, and a $\mathrm{Mo}^{(\mathrm{B})}$ $3 \mathrm{~d}$ doublet at $\mathrm{BE}$ of $229.3-232.45 \mathrm{eV}$. The $\mathrm{Mo}^{(\mathrm{A})}$ doublet was attributed to $\mathrm{Mo}^{5+}$ chemical state ${ }^{28}$ or $\mathrm{Mo}^{4+}$ as in molybdenum oxysulfide $\operatorname{MoS}_{x} \mathrm{O}_{y} \cdot{ }^{30,37} \mathrm{The} \mathrm{Mo}^{(\mathrm{B})}$ doublet was attributed to $\mathrm{Mo}^{4+}$ as in $\mathrm{MoS}_{2}$ or $\mathrm{MoS}_{3}{ }^{37}$ Both Mo and $\mathrm{S}$ appeared in mixed chemical states and in good agreement with reported XPS spectra of amorphous $\mathrm{MoS}_{3} \cdot{ }^{36}$ As opposed to the XPS spectra of $\operatorname{MoS}_{x} /$ GCNT/CP, typical XPS spectra of crystalline $\mathrm{MoS}_{2}$ only show $\mathrm{Mo}^{(\mathrm{B})}$ and $\mathrm{S}^{(\mathrm{B})}$ chemical states. Using XPS spectra of crystalline $\mathrm{MoS}_{2}$ as references, the stoichiometry of $\mathrm{MoS}_{x}$ was found as $\mathrm{Mo}: \mathrm{S}=1: 3.09$.

The amorphous nature of $\operatorname{MoS}_{x}$ was further examined with Raman spectroscopy and XRD. Fig. 3a shows the Raman spectrum of the $\mathrm{MoS}_{x} / \mathrm{GCNT} / \mathrm{CP}$ electrode. As opposed to crystalline
$\mathrm{MoS}_{2}$ of which the Raman spectrum typically shows characteristic peaks $\mathrm{E}_{2 \mathrm{~g}}^{1}$ and $\mathrm{A}_{1 \mathrm{~g}}$, the Raman spectrum of the as-deposited $\operatorname{MoS}_{x}$ showed broad peaks with Raman shift of between 200 and
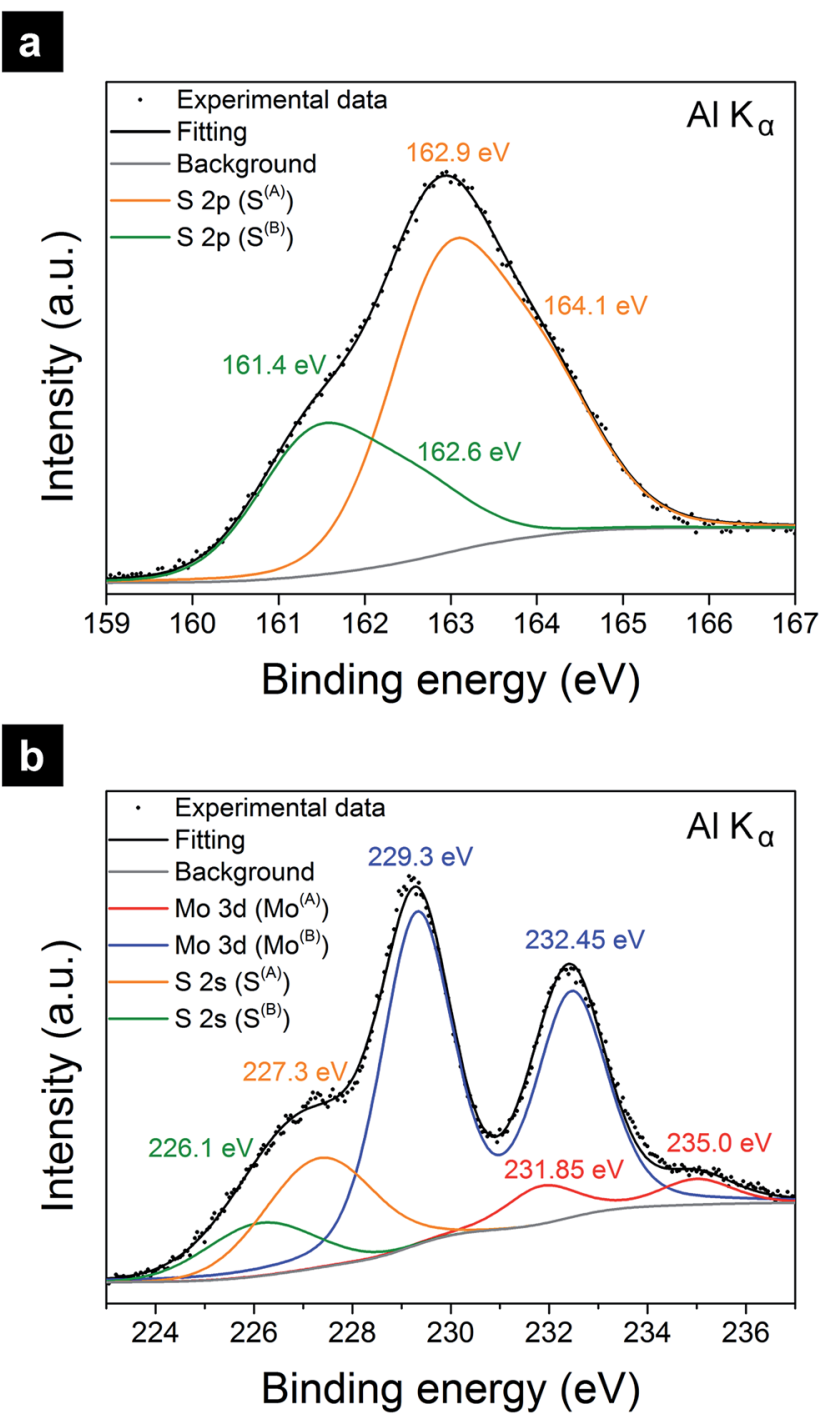

Fig. 2 Chemical analysis of the MoS $_{x} /$ GCNT/CP electrode with XPS in (a) $S 2 p$, and (b) Mo $3 d-S 2 s$ regions. 
a
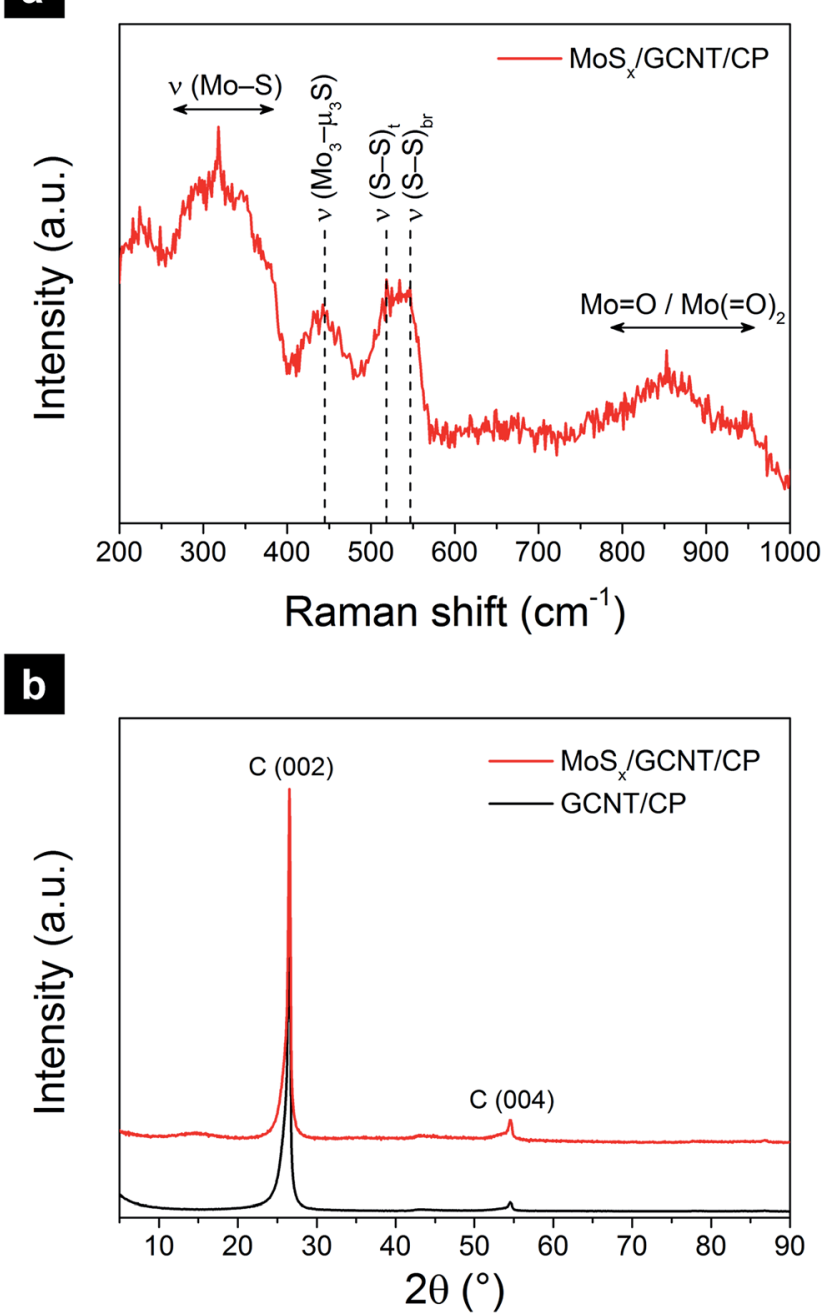

Fig. 3 Crystallinity analyses of the $M_{0} S_{x} / G C N T / C P$ electrode with (a) Raman spectroscopy, and (b) XRD.

$1000 \mathrm{~cm}^{-1}$. The spectrum was in good agreement with previously reported Raman spectra of amorphous molybdenum sulfide materials. ${ }^{36,38,39}$ The Raman peaks at $\sim 550$ and $\sim 520$ $\mathrm{cm}^{-1}$ were assigned to the vibration modes of bridging $\mathrm{S}_{2}{ }^{2-}$ $\left(\nu(\mathrm{S}-\mathrm{S})_{\mathrm{br}}\right)$ and terminal $\mathrm{S}_{2}{ }^{2-}\left(\nu(\mathrm{S}-\mathrm{S})_{\mathrm{t}}\right)$, respectively. ${ }^{39}$ The Raman peaks at $\sim 270-380 \mathrm{~cm}^{-1}$ and $\sim 445 \mathrm{~cm}^{-1}$ were assigned to molybdenum sulfide vibration modes of $\nu(\mathrm{Mo}-\mathrm{S})$ and $\nu\left(\mathrm{Mo}_{3}{ }^{-}\right.$ $\mu_{3} \mathrm{~S}$ ), respectively. ${ }^{39}$ The pronounced vibration modes of bridging and terminal $\mathrm{S}_{2}{ }^{2-}$ as well as apical sulfide center were characteristic for the amorphous nature of the as-deposited $\operatorname{MoS}_{x}$. The Raman peaks at $\sim 800-950 \mathrm{~cm}^{-1}$ was assigned to molybdenum oxide defects, ${ }^{39}$ suggesting the possible presence of molybdenum oxysulfide $\operatorname{MoS}_{x} \mathrm{O}_{y}$. The possible presence of $\mathrm{MoS}_{x} \mathrm{O}_{y}$ was also evident by the $\mathrm{Mo}^{(\mathrm{A})}$ state in the XPS analysis. To further probe the amorphous nature of $\operatorname{MoS}_{x}$, XRD measurements were performed on the GCNT/CP and $\operatorname{MoS}_{x} /$ GCNT/CP electrodes. The resulting XRD spectra are shown in Fig. 3b. Both spectra showed sharp and strong characteristic peaks of graphitic carbon (002) plane, and therefore indicated

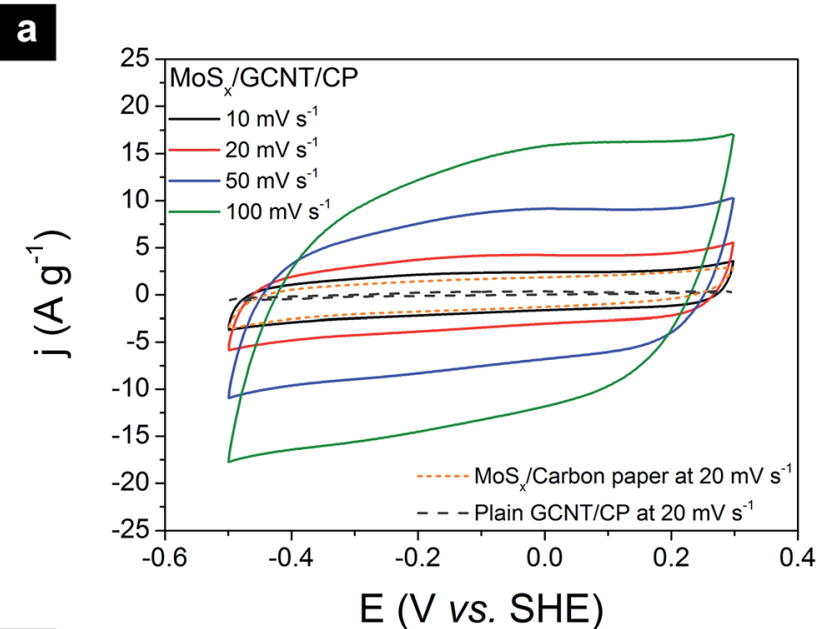

b

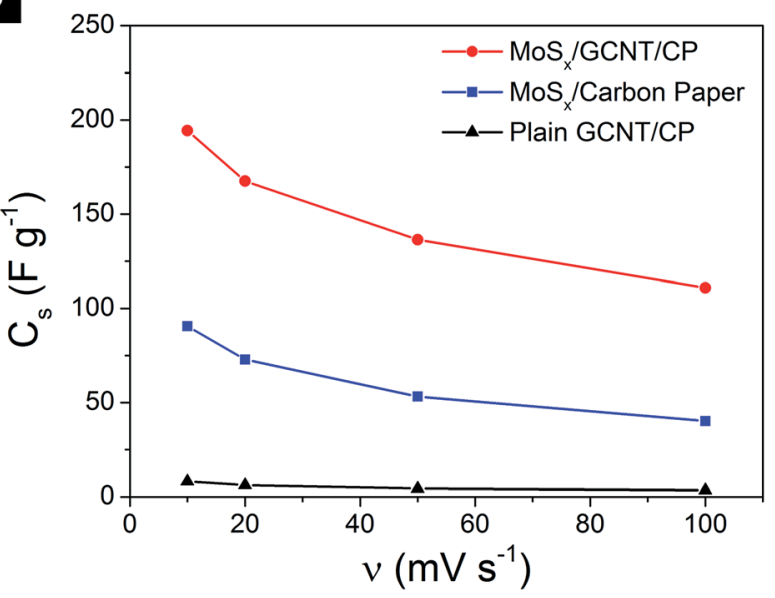

Fig. 4 Supercapacitive performance of electrodes assessed by cyclic voltammetry. (a) Cyclic voltammograms of the MoS $/$ GCNT/CP electrode at different scan rates from 10 to $100 \mathrm{mV} \mathrm{s}^{-1}$, of the bare GCNT/ $\mathrm{CP}$ support and $\mathrm{MoS}_{x} /$ carbon paper electrode at $20 \mathrm{mV} \mathrm{s}^{-1}$ scan rate. (b) The dependence of electrode specific capacitance on scan rate.

the high crystallinity of the GCNT/CP support. The XRD spectrum of $\mathrm{MoS}_{x} / \mathrm{GCNT} / \mathrm{CP}$ did not show any strong additional characteristic peaks that could otherwise suggest the presence of crystalline $\mathrm{MoS}_{2}$, except very weak and broad diffraction bands of $2 \theta$ from $10^{\circ}$ to $20^{\circ}$ and from $30^{\circ}$ to $40^{\circ}$. The XRD result further indicated the amorphous nature of the deposited $\operatorname{MoS}_{x}$.

\subsection{Supercapacitive properties of the $\operatorname{MoS}_{x} / \mathrm{GCNT} / \mathrm{CP}$ electrode}

The supercapacitive properties of $\mathrm{MoS}_{x} / \mathrm{GCNT} / \mathrm{CP}$ electrode were assessed with cyclic voltammetry and galvanostatic charge/ discharge. Fig. 4a shows the cyclic voltammograms of $\operatorname{MoS}_{x} /$ GCNT/CP electrode obtained at scan rates of 10, 20, 50, and $100 \mathrm{mV} \mathrm{s}^{-1}$. For reference purposes, the cyclic voltammograms of bare GCNT/CP support and of $\mathrm{MoS}_{x} /$ carbon paper were also recorded. However, for a clearer presentation, only the cyclic voltammograms obtained at the scan rate of $20 \mathrm{mV} \mathrm{s}^{-1}$ were plotted for these two reference samples. It should be noted that, while the bare GCNT/CP sample did not contain any $\operatorname{MoS}_{x}$, in 
order to present the contribution of the GCNT/CP support to the overall capacitance of $\mathrm{MoS}_{x} / \mathrm{GCNT} / \mathrm{CP}$, the cyclic voltammograms of the bare GCNT/CP were also normalized for a hypothetical $\mathrm{MoS}_{x}$ loading of $3 \mathrm{mg} \mathrm{cm}{ }^{-2}$. The cyclic voltammograms of $\mathrm{MoS}_{x} / \mathrm{GCNT} / \mathrm{CP}$ appeared quasi-rectangular. The quasirectangular shape was retained even at a high scan rate of $100 \mathrm{mV} \mathrm{s}^{-1}$, which suggested a good high-scan-rate capacitive performance of the $\mathrm{MoS}_{x} / \mathrm{GCNT} / \mathrm{CP}$ electrode. The $\mathrm{MoS}_{x} / \mathrm{GCNT} /$ $\mathrm{CP}$ electrode showed much higher current density $j$ than that of the GCNT/CP support and of the $\mathrm{MoS}_{x} /$ carbon paper electrode.

The gravimetric specific capacitance of electrodes was calculated as following:

$$
C_{\mathrm{s}}=\frac{\int j(E) \mathrm{d} E}{2 v \Delta E}
$$

in which $C_{\mathrm{s}}$ is the gravimetric specific capacitance of electrode (in $\left.\mathrm{F}_{\mathrm{MoS}_{x}}{ }^{-1}\right), j(E)$ is the instantaneous gravimetric current density (in $\mathrm{A}_{\mathrm{Mos}_{x}}{ }^{-1}$ ), $v$ is the scan rate (in $\mathrm{V} \mathrm{s}^{-1}$ ), $\Delta E$ is the scanned potential window of $0.8 \mathrm{~V} \cdot \int j(E) \mathrm{d} E$ is the integral area enclosed by the cyclic voltammograms.

The calculated $C_{\mathrm{s}}$ of electrodes based on cyclic voltammetry are summarized in Fig. 4b. The $\operatorname{MoS}_{x} / \mathrm{GCNT} / \mathrm{CP}$ electrode demonstrated a capacitance of $194 \mathrm{~F} \mathrm{~g}^{-1}$ at the scan rate of $10 \mathrm{mV} \mathrm{s}^{-1}$ and retained a capacitance of $111 \mathrm{~F} \mathrm{~g}^{-1}$ at a high scan rate of $100 \mathrm{mV} \mathrm{s}^{-1}$. The capacitive contribution from the GCNT/ CP support to the total capacitance of the $\mathrm{MoS}_{x} / \mathrm{GCNT} / \mathrm{CP}$ electrode was minimal (e.g. $8.2 \mathrm{~F} \mathrm{~g}^{-1}$ at the scan rate of $10 \mathrm{mV}$ $\mathrm{s}^{-1}$ ). $\mathrm{MoS}_{x} /$ carbon paper showed much lower capacitance than that of the $\operatorname{MoS}_{x} / \mathrm{GCNT} / \mathrm{CP}$ electrode. $\mathrm{MoS}_{x} /$ carbon paper showed a capacitance of $91 \mathrm{~F} \mathrm{~g}^{-1}$ at the scan rate of $10 \mathrm{mV} \mathrm{s}^{-1}$ and a capacitance of $40 \mathrm{~F} \mathrm{~g}^{-1}$ at the scan rate of $100 \mathrm{mV} \mathrm{s}^{-1}$. The reported capacitance values were 2-3 times lower than those of the $\operatorname{MoS}_{x}$ deposited on the high surface area GCNT/CP support. In this work, both $\operatorname{MoS}_{x} / \mathrm{GCNT} / \mathrm{CP}$ and $\operatorname{MoS}_{x} /$ carbon paper samples were prepared using the same $\operatorname{MoS}_{x}$ deposition protocol. As such, the specific capacitance enhancement in the $\mathrm{MoS}_{x} / \mathrm{GCNT} / \mathrm{CP}$ as compared to $\mathrm{MoS}_{x} /$ carbon paper was attributed to the enhanced dispersion of $\operatorname{MoS}_{x}$, better access to the electrolyte, and more efficient electron transport, resulted from a much higher specific surface area of the GCNT/CP support. Comparisons of the specific surface area of GCNT/CP and carbon paper supports have been reported in detail in our previous work. ${ }^{33}$ As reported in our previous work, the GCNT/CP support had $\approx 50$ times higher roughness factor (the ratio between the microscopic extended surface area and the electrode projected surface area) compared to the simple carbon paper support. ${ }^{33}$ The deposition of $\operatorname{MoS}_{x}$ on a high surface area conductive support demonstrated this to be an effective method to enhance the electrode capacitance.

The specific capacitance of $\mathrm{MoS}_{x}$ can also be characterized with galvanostatic charge/discharge curves, as shown in Fig. 5 . Fig. 5a shows the galvanostatic charge/discharge curves of the $\mathrm{MoS}_{x} / \mathrm{GCNT} / \mathrm{CP}$ electrode, measured at different charge/ discharge rates from 0.67 to $16.7 \mathrm{~A} \mathrm{~g}^{-1}$. The charge/discharge curves were quasi-triangular at the low charge/discharge rate of $0.67 \mathrm{~A} \mathrm{~g}^{-1}$ and became more symmetric and triangular at

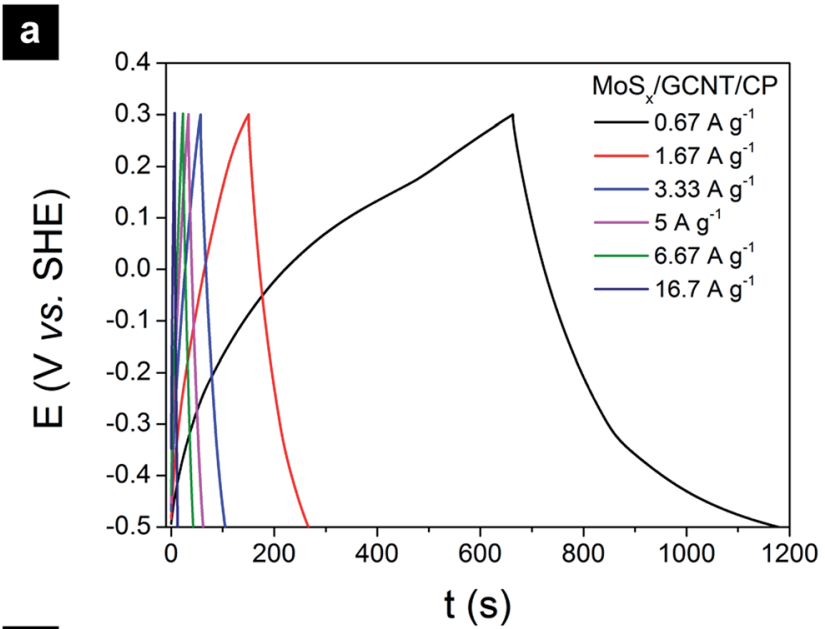

b
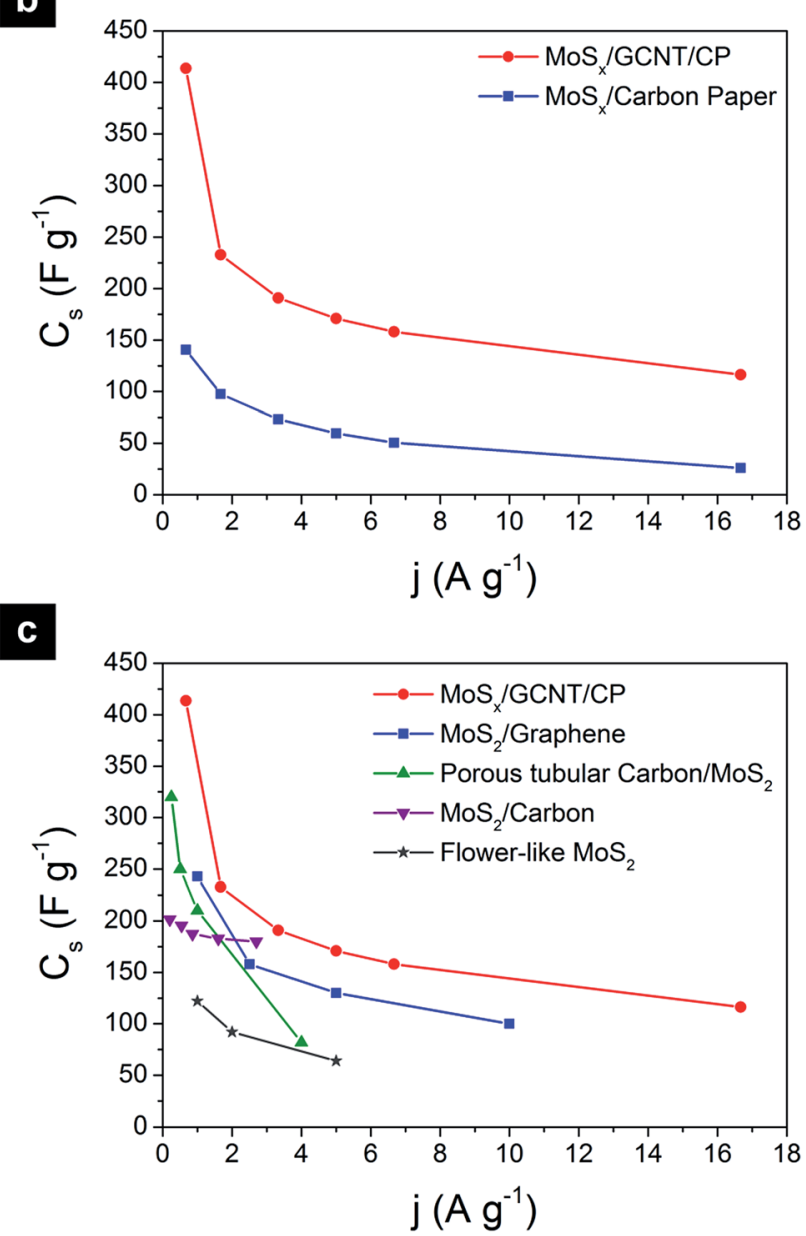

Fig. 5 Supercapacitive performance of electrodes assessed by galvanostatic charge/discharge curves. (a) Charge/discharge curves of the $\mathrm{MOS}_{x} / \mathrm{GCNT/CP}$ electrode obtained at different charge/discharge rates from 0.67 to $16.7 \mathrm{~A} \mathrm{~g}^{-1}$. (b) The dependence of electrode specific capacitance on charge/discharge rate. (c) A comparison of the discharge capacitance of $\mathrm{MoS}_{x} / \mathrm{GCNT/CP}$ against crystalline $\mathrm{MoS}_{2}-$ based electrode materials reported in the literature.

higher charge/discharge rates. The gravimetric specific discharge capacitance of $\mathrm{MoS}_{x} / \mathrm{GCNT} / \mathrm{CP}$ based on the charge/ discharge curves was calculated as following: 


$$
C_{\mathrm{s}}=\frac{j \Delta t}{\Delta E}
$$

in which $C_{\mathrm{s}}$ is the gravimetric specific discharge capacitance of electrode (in $\mathrm{F} \mathrm{g}_{\mathrm{Mos}_{x}}^{-1}$ ), $j$ is the gravimetric galvanostatic discharge rate (in $\mathrm{A} \mathrm{g}_{\mathrm{Mos}_{x}}{ }^{-1}$ ), $\Delta t$ is the discharge time (in s), $\Delta E$ is the charge/discharge potential window of $0.8 \mathrm{~V}$.

The calculated $C_{\mathrm{s}}$ of electrodes based on galvanostatic charge/ discharge curves are summarized in Fig. 5b. The $\operatorname{MoS}_{x} /$ GCNT/CP electrode demonstrated a remarkable discharge capacitance of $414 \mathrm{~F} \mathrm{~g}^{-1}$ at the discharge rate of $0.67 \mathrm{~A} \mathrm{~g}^{-1}$. The $\mathrm{MoS}_{x} / \mathrm{GCNT} / \mathrm{CP}$ electrode retained a high discharge capacitance of $116 \mathrm{~F} \mathrm{~g}^{-1}$ even at a high discharge rate of $16.7 \mathrm{~A} \mathrm{~g}^{-1}$. Similar to the cyclic voltammetry experiments, the $\mathrm{MoS}_{x} /$ carbon paper electrode once again showed much lower specific capacitance than that of the $\mathrm{MoS}_{x} / \mathrm{GCNT} / \mathrm{CP}$ electrode, with $C_{\mathrm{S}}$ of 140 and $26 \mathrm{~F} \mathrm{~g}^{-1}$ at discharge rates of 0.67 and $16.7 \mathrm{~A} \mathrm{~g}^{-1}$, respectively. A 3-4.5 fold enhancement factor in specific capacitance was demonstrated by $\mathrm{MoS}_{x} / \mathrm{GCNT} / \mathrm{CP}$ as compared to $\mathrm{MoS}_{x} /$ carbon paper. The strongly enhanced specific capacitance of $\operatorname{MoS}_{x}$ when being deposited on a high surface area support was once again demonstrated through the galvanostatic charge/discharge experiments.

With the specific capacitance of $414 \mathrm{~F} \mathrm{~g}^{-1}$ obtained at the discharge rate of $0.67 \mathrm{~A} \mathrm{~g}^{-1}$, the $\mathrm{MoS}_{x} / \mathrm{GCNT} / \mathrm{CP}$ electrode demonstrated a significant improvement in specific capacitance over that of carbon-based supercapacitor electrode materials. ${ }^{\mathbf{4 0}, 41}$ Although the demonstrated capacitance was lower than the best-in-class capacitance demonstrated by hydrous ruthenium oxide $\left(\mathrm{RuO}_{2} \cdot x \mathrm{H}_{2} \mathrm{O}\right),{ }^{\mathbf{4 2 , 4 3}} \mathrm{MoS}_{x}$ has the advantages of low material cost and simple room-temperature synthesis. The high cost of precious metal in $\mathrm{RuO}_{2}$ has been preventing the material from practical applications despite the high supercapacitive performance. Fig. 5 c shows a comparison of discharge capacitance of the $\mathrm{MoS}_{x} / \mathrm{GCNT} / \mathrm{CP}$ electrode with examples of crystalline $\mathrm{MoS}_{2}$-based electrode materials reported in the literature, including $\mathrm{MoS}_{2} /$ graphene, ${ }^{17}$ porous tubular carbon/ $\mathrm{MoS}_{2},{ }^{13} \mathrm{MoS}_{2} /$ carbon composite, ${ }^{44}$ and flowerlike $\mathrm{MoS}_{2}$ nanospheres. ${ }^{45}$ The $\mathrm{MoS}_{x} / \mathrm{GCNT} / \mathrm{CP}$ electrode consistently showed better high-discharge-rate capacitance than the crystalline $\mathrm{MoS}_{2}$ materials, in which their capacitances decreased rapidly at higher discharge current densities.
The higher capacitance at high discharge rates of the $\operatorname{MoS}_{x}$ l GCNT/CP electrode could be due to the faster ion diffusion in $\mathrm{MoS}_{x}$ as compared to crystalline $\mathrm{MoS}_{2}$ and/or the good electron and electrolytic ion transport in the $\mathrm{MoS}_{x} / \mathrm{GCNT} / \mathrm{CP}$ electrode.

Table 1 provides a comparison for the specific capacitance of the $\mathrm{MoS}_{x} / \mathrm{GCNT} / \mathrm{CP}$ electrode against other representative supercapacitor materials reported in the literature. Due to the large variety of specific material approaches, the specific capacitance of materials other than $\mathrm{MoS}_{x}$ and $\mathrm{MoS}_{2}$-based materials were presented by the typical range of specific capacitance reported in the literature.

Coulombic efficiency during charge/discharge cycles of the $\mathrm{MoS}_{x} / \mathrm{GCNT} / \mathrm{CP}$ electrode started at relatively modest values of between $77 \%$ and $87 \%$-depending on the charge/discharge rate-in the first charge/discharge cycle. However, the coulombic efficiency of the electrode quickly reached above 97\% after 10 charge/discharge cycles, and above 99\% after 25 charge/discharge cycles at $5 \mathrm{~A} \mathrm{~g}^{-1}$.

For a better understanding of the electrode interfacial properties, EIS was performed at $-0.1 \mathrm{~V} v$ s. SHE. EIS spectra of the $\mathrm{MoS}_{x} / \mathrm{GCNT} / \mathrm{CP}$ and $\mathrm{MoS}_{x} /$ carbon paper electrodes were analyzed with equivalent circuit fitting. The EIS spectra and fitting curves of the electrodes are shown in Fig. 6. Fig. 6a and b show the EIS spectra and fitting curves presented in Nyquist plot and Bode plots, respectively. The Nyquist plots of the $\operatorname{MoS}_{x} /$ GCNT/CP and $\operatorname{MoS}_{x} /$ carbon paper electrodes showed nearly vertical curves, which suggested nearly idealistic capacitive behaviors of the electrodes. The equivalent circuit used for all fittings is presented in Fig. 6c. In this circuit, $L_{\text {cab }}$ represents a parasitic inductance due to connecting cables or instruments. A minor inductive response was observed at the high frequency of $\sim 10^{4} \mathrm{~Hz} . R_{\mathrm{ohm}}$ represents the ohmic resistance contributed by the uncompensated electrolyte resistance and internal resistance of electrode. $R_{\text {con }}$ and $C_{\text {con }}$ represent contact resistance and contact capacitance, respectively. The contact resistance and contact capacitance account for the small arc at high frequency range of $\sim 10^{3} \mathrm{~Hz} . R_{\mathrm{ct}}, W$, and $\mathrm{CPE}_{\mathrm{dl}}$ represent the charge transfer resistance, Warburg diffusion impedance, and electrical double layer capacitance constant phase element,

Table 1 Comparison for the specific capacitance of the $\operatorname{MoS}_{x} / \mathrm{GCNT/CP}$ electrode against the reported literature

\begin{tabular}{lll} 
& $\begin{array}{l}\text { Typical range of specific } \\
\text { capacitance }\left(\mathrm{F} \mathrm{g}^{-1}\right)\end{array}$ & References \\
\hline Activated carbon & $115-340$ & $46-48$ \\
Purified CNTs & $20-80$ & 49 and 50 \\
Graphene, reduced graphene oxide & $135-205$ & 2 and 51 \\
Hydrous RuO & $720-1300$ & 42 and $52-54$ \\
$\mathrm{MnO}_{2}$ & $195-350$ \\
$\mathrm{Conductive} \mathrm{polymers}_{\mathrm{MoS}_{x} / \mathrm{GCNT} / \mathrm{CP}}$ & $230-1030$ \\
$\mathrm{MoS}_{2} /$ graphene & $414\left(\right.$ at $\left.0.67 \mathrm{~A} \mathrm{~g}^{-1}\right)$ & $55-57$ \\
$\mathrm{Porous}_{\text {tubular carbon/MoS }}$ & $243\left(\right.$ at $\left.1 \mathrm{~A} \mathrm{~g}^{-1}\right)$ & $58-63$ \\
$\mathrm{MoS}_{2} /$ carbon & $250\left(\right.$ at $\left.0.5 \mathrm{~A} \mathrm{~g}^{-1}\right)$ & 17 \\
Flower-like MoS & $195\left(\right.$ at $\left.0.54 \mathrm{~A} \mathrm{~g}^{-1}\right)$ & 13 \\
$1 \mathrm{~T}-\mathrm{MoS}_{2}$ & $122\left(\right.$ at $\left.1 \mathrm{~A} \mathrm{~g}^{-1}\right)$ & 44 \\
& $\sim 100\left(\right.$ at $\left.20 \mathrm{mV} \mathrm{s}^{-1}\right)$ &
\end{tabular}


a

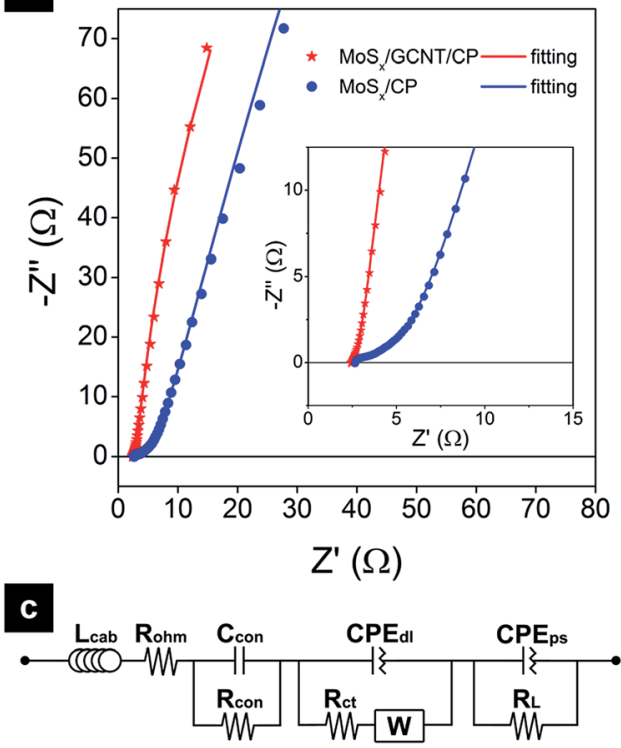

b

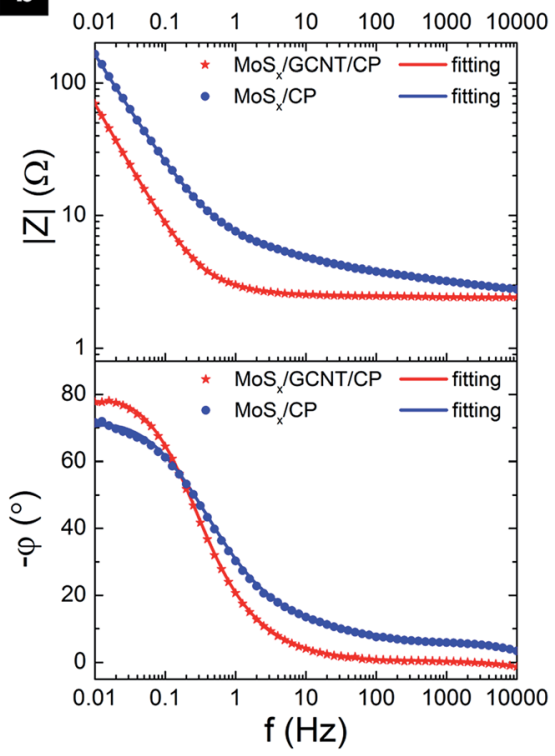

Fig. 6 EIS spectra and equivalent circuit fittings of the MoS $_{x} /$ GCNT/CP and MoS $_{x} /$ carbon paper electrodes presented in (a) Nyquist plot with (inset) the closed up view at high frequency region, and (b) Bode plots. (c) Equivalent circuit used for all fittings in (a, b).

respectively. $R_{\mathrm{L}}$ and $\mathrm{CPE}_{\mathrm{ps}}$ are leak resistance and pseudocapacitance constant phase element, respectively. Equivalent circuit fitting results showed that $\mathrm{MoS}_{x} / \mathrm{GCNT} / \mathrm{CP}$ demonstrated a much faster charge transfer kinetic $\left(R_{\mathrm{ct}}=0.352 \Omega \mathrm{cm}^{2}\right)$ as compared to $\operatorname{MoS}_{x}$ deposited on carbon paper $\left(R_{\mathrm{ct}}=4.850 \Omega\right.$ $\mathrm{cm}^{2}$ ). The enhancement in charge transfer kinetic (13.8 times smaller $R_{\mathrm{ct}}$ ) was most likely attributed to the effective material dispersion and efficient electron transport towards $\mathrm{MoS}_{x}$ provided by the GCNT/CP support. Similar enhancement in kinetic was also demonstrated in the HER application of these materials. ${ }^{33}$ The equivalent pseudo-capacitance $\mathrm{CPE}_{\mathrm{ps}}$ of $\mathrm{MoS}_{x} /$

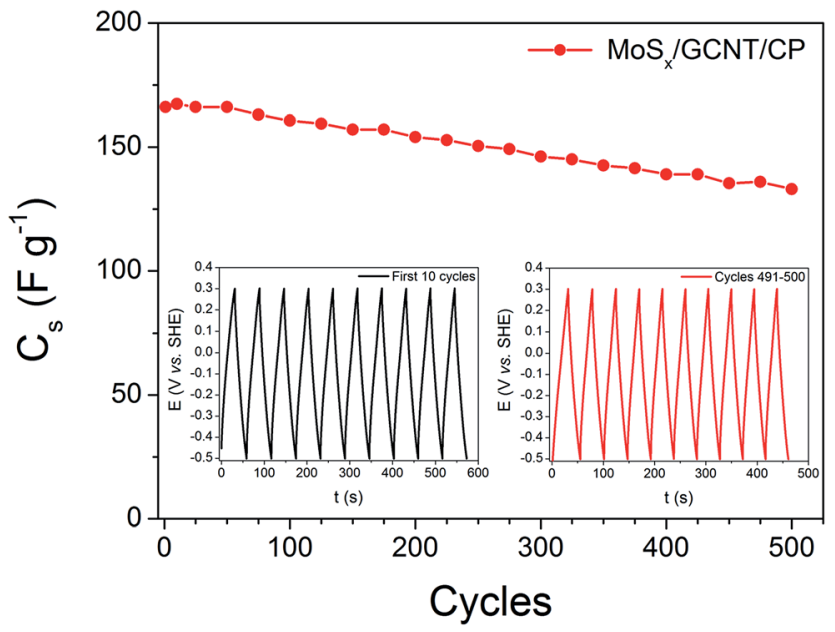

Fig. 7 Degradation test of the $\mathrm{MOS}_{x} / \mathrm{GCNT/CP}$ electrode performed with 500 charge/discharge cycles at $5 \mathrm{~A} \mathrm{~g}^{-1}$, showing the degradation of electrode specific capacitance during the test, with (insets) plots of the first and last 10 charge/discharge cycles (time axes $t$ are referenced to the beginning of each 10 -cycle set).
GCNT/CP $\left(157 \mathrm{~F} \mathrm{~g}^{-1}\right)$ was almost twice that of $\mathrm{MoS}_{x} /$ carbon paper $\left(80 \mathrm{~F} \mathrm{~g}^{-1}\right)$.

Beside a high capacitance, the electrochemical stability of supercapacitor electrode materials is also of great interest. The stability of the $\mathrm{MoS}_{x} / \mathrm{GCNT} / \mathrm{CP}$ electrode was evaluated by a degradation test performed with 500 galvanostatic charge/ discharge cycles at a high charge/discharge current density of $5 \mathrm{~A} \mathrm{~g}^{-1}$. The degradation of the electrode discharge capacitance during the degradation test is reported in Fig. 7. Fig. 7 shows that the discharge capacitance of the electrode slowly degraded during the test with an $80 \%$ retention of capacitance after 500 charge/discharge cycles. It should be noted that the degradation test was performed at a high charge/discharge rate of $5 \mathrm{~A} \mathrm{~g}^{-1}$. $\mathrm{A}$ high charge/discharge current can potentially degrade the electrode faster than a slow charge/discharge rate due to the quick ion insertion into and extraction from the electrode active materials. Capacitance degradation is commonly found in pseudo-capacitive active materials. Although further optimizations of the electrode will need to be pursued, we consider an $80 \%$ capacitance retention at a high charge/discharge rate of $5 \mathrm{~A}$ $\mathrm{g}^{-1}$ a good electrochemical stability demonstrated by the $\operatorname{MoS}_{x} /$ GCNT/CP electrode.

\section{Conclusions}

In this work, we report the application of $\operatorname{MoS}_{x}$ as the main active material for supercapacitor electrodes. $\mathrm{MoS}_{x}$ was deposited onto a high surface area conductive electrode of GCNT/CP at room temperature. $\operatorname{MoS}_{x}$ was successfully deposited on the GCNT hybrids and formed intimate contacts with the conductive GCNT/CP support. The $\mathrm{MoS}_{x} / \mathrm{GCNT} / \mathrm{CP}$ composite electrode showed high specific capacitance even at high discharge rates. The deposition of $\operatorname{MoS}_{x}$ on a conductive and high specific 
surface area support demonstrated this to be an effective method to enhance the supercapacitive performance of $\operatorname{MoS}_{x}$. Together with the good electrochemical stability, the $\operatorname{MoS}_{x} /$ GCNT/CP electrode was a promising electrode material candidate for supercapacitor applications.

\section{Acknowledgements}

K. C. P., D. H. C. C. and A. T. S. W. acknowledge the support from National University of Singapore (NUS) (Grants number: R284-000-142-112, R284-000-120-281, and R-144-000-321-112). K. C. P. acknowledges NUS Graduate School for Integrative Sciences and Engineering for the NGS Scholarship. K. C. P. acknowledges Mr Wong How Kwong, Dr Zheng Minrui and Ms Pang Teng Jar for their assistance with XPS, Raman spectroscopy and XRD, respectively.

\section{References}

1 G. Wang, L. Zhang and J. Zhang, Chem. Soc. Rev., 2012, 41, 797-828.

2 Y. Wang, Z. Shi, Y. Huang, Y. Ma, C. Wang, M. Chen and Y. Chen, J. Phys. Chem. C, 2009, 113, 13103-13107.

3 K.-J. Huang, L. Wang, J.-Z. Zhang, L.-L. Wang and Y.-P. Mo, Energy, 2014, 67, 234-240.

4 J. Gamby, P. L. Taberna, P. Simon, J. F. Fauvarque and M. Chesneau, J. Power Sources, 2001, 101, 109-116.

5 C. Liu, Z. Yu, D. Neff, A. Zhamu and B. Z. Jang, Nano Lett., 2010, 10, 4863-4868.

6 E. Frackowiak, K. Metenier, V. Bertagna and F. Beguin, Appl. Phys. Lett., 2000, 77, 2421-2423.

7 Y. Hou, Y. Cheng, T. Hobson and J. Liu, Nano Lett., 2010, 10, 2727-2733.

8 H. Jiang, J. Ma and C. Li, Adv. Mater., 2012, 24, 4197-4202.

9 R. R. Salunkhe, K. Jang, S.-w. Lee, S. Yu and H. Ahn, J. Mater. Chem., 2012, 22, 21630-21635.

10 H. Jiang, T. Zhao, C. Li and J. Ma, J. Mater. Chem., 2011, 21, 3818-3823.

11 S. Ratha and C. S. Rout, ACS Appl. Mater. Interfaces, 2013, 5, 11427-11433.

12 M. Acerce, D. Voiry and M. Chhowalla, Nat. Nanotechnol., 2015, 10, 313-318.

13 B. Hu, X. Qin, A. M. Asiri, K. A. Alamry, A. O. Al-Youbi and X. Sun, Electrochim. Acta, 2013, 100, 24-28.

14 J. M. Soon and K. P. Loh, Electrochem. Solid-State Lett., 2007, 10, A250-A254.

15 E. G. da Silveira Firmiano, A. C. Rabelo, C. J. Dalmaschio, A. N. Pinheiro, E. C. Pereira, W. H. Schreiner and E. R. Leite, Adv. Energy Mater., 2014, 4, DOI: 10.1002/ aenm.201301380.

16 G. Sun, X. Zhang, R. Lin, J. Yang, H. Zhang and P. Chen, Angew. Chem., Int. Ed., 2015, 54, 4651-4656.

17 K.-J. Huang, L. Wang, Y.-J. Liu, Y.-M. Liu, H.-B. Wang, T. Gan and L.-L. Wang, Int. J. Hydrogen Energy, 2013, 38, 1402714034.

18 A. Ramadoss, T. Kim, G.-S. Kim and S. J. Kim, New J. Chem., 2014, 38, 2379-2385.
19 Y. Zhang, W. Sun, X. Rui, B. Li, H. T. Tan, G. Guo, S. Madhavi, Y. Zong and Q. Yan, Small, 2015, 11, 3694-3702. 20 K.-J. Huang, J.-Z. Zhang and J.-L. Cai, Electrochim. Acta, 2015, 180, 770-777.

21 X. Liu, J.-Z. Zhang, K.-J. Huang and P. Hao, Chem. Eng. J., 2016, 302, 437-445.

22 K.-J. Huang, J.-Z. Zhang, Y.-L. Jia, K. Xing and Y.-M. Liu, J. Alloys Compd., 2015, 641, 119-126.

23 K.-J. Huang, J.-Z. Zhang and Y. Fan, J. Alloys Compd., 2015, 625, 158-163.

24 Y. Miki, D. Nakazato, H. Ikuta, T. Uchida and M. Wakihara,J. Power Sources, 1995, 54, 508-510.

25 M. Bouroushian, Electrochemistry of Metal Chalcogenides, Springer-Verlag Berlin Heidelberg, Berlin Heidelberg, 1st edn, 2010.

26 M. Pumera, Z. Sofer and A. Ambrosi, J. Mater. Chem. A, 2014, 2, 8981-8987.

27 D. J. Li, U. N. Maiti, J. Lim, D. S. Choi, W. J. Lee, Y. Oh, G. Y. Lee and S. O. Kim, Nano Lett., 2014, 14, 1228-1233.

28 J. D. Benck, Z. Chen, L. Y. Kuritzky, A. J. Forman and T. F. Jaramillo, ACS Catal., 2012, 2, 1916-1923.

29 D. Merki, S. Fierro, H. Vrubel and X. Hu, Chem. Sci., 2011, 2, 1262-1267.

30 C. G. Morales-Guio and X. Hu, Acc. Chem. Res., 2014, 47, 2671-2681.

31 K.-C. Pham, D. H. C. Chua, D. S. McPhail and A. T. S. Wee, ECS Electrochem. Lett., 2014, 3, F37-F40.

32 K.-C. Pham, D. S. McPhail, C. Mattevi, A. T. S. Wee and D. H. C. Chua, J. Electrochem. Soc., 2016, 163, F255-F263.

33 K.-C. Pham, Y.-H. Chang, D. S. McPhail, C. Mattevi, A. T. S. Wee and D. H. C. Chua, ACS Appl. Mater. Interfaces, 2016, 8, 5961-5971.

34 K. C. Pham, NUS-ICL Joint Ph.D. (NGS) thesis, National University of Singapore, 2016.

35 T. A. J. Loh, Y. Hu, K. C. Pham, Z. Tan and D. H. C. Chua, IEEE 16th International Conference on Nanotechnology (IEEENANO), 2016, pp. 899-900.

36 T. Weber, J. C. Muijsers and J. W. Niemantsverdriet, J. Phys. Chem., 1995, 99, 9194-9200.

37 H. Vrubel and X. Hu, ACS Catal., 2013, 3, 2002-2011.

38 C. H. Chang and S. S. Chan, J. Catal., 1981, 72, 139-148.

39 P. D. Tran, T. V. Tran, M. Orio, S. Torelli, Q. D. Truong, K. Nayuki, Y. Sasaki, S. Y. Chiam, R. Yi, I. Honma, J. Barber and V. Artero, Nat. Mater., 2016, 15, 640-646.

40 L. L. Zhang and X. S. Zhao, Chem. Soc. Rev., 2009, 38, 25202531.

41 L. L. Zhang, R. Zhou and X. S. Zhao, J. Mater. Chem., 2010, 20, 5983-5992.

42 C.-C. Hu, K.-H. Chang, M.-C. Lin and Y.-T. Wu, Nano Lett., 2006, 6, 2690-2695.

43 R. K. Das, B. Liu, J. R. Reynolds and A. G. Rinzler, Nano Lett., 2009, 9, 677-683.

44 L.-Q. Fan, G.-J. Liu, C.-Y. Zhang, J.-H. Wu and Y.-L. Wei, Int. J. Hydrogen Energy, 2015, 40, 10150-10157.

$45 \mathrm{X}$. Zhou, B. Xu, Z. Lin, D. Shu and L. Ma, J. Nanosci. Nanotechnol., 2014, 14, 7250-7254. 
46 J. Jiang, L. Zhang, X. Wang, N. Holm, K. Rajagopalan, F. Chen and S. Ma, Electrochim. Acta, 2013, 113, 481-489.

47 X. Du, W. Zhao, Y. Wang, C. Wang, M. Chen, T. Qi, C. Hua and M. Ma, Bioresour. Technol., 2013, 149, 31-37.

48 A. Thambidurai, J. K. Lourdusamy, J. V. John and S. Ganesan, Korean J. Chem. Eng., 2014, 31, 268-275.

49 A. G. Pandolfo and A. F. Hollenkamp, J. Power Sources, 2006, 157, 11-27.

50 S. Talapatra, S. Kar, S. K. Pal, R. Vajtai, L. Ci, P. Victor, M. M. Shaijumon, S. Kaur, O. Nalamasu and P. M. Ajayan, Nat. Nanotechnol., 2006, 1, 112-116.

51 M. D. Stoller, S. Park, Y. Zhu, J. An and R. S. Ruoff, Nano Lett., 2008, 8, 3498-3502.

52 J. P. Zheng, P. J. Cygan and T. R. Jow, J. Electrochem. Soc., 1995, 142, 2699-2703.

53 Y.-Z. Zheng, H.-Y. Ding and M.-L. Zhang, Thin Solid Films, 2008, 516, 7381-7385.

54 J. P. Zheng and T. R. Jow, J. Electrochem. Soc., 1995, 142, L6L8.
55 C.-C. Hu and T.-W. Tsou, Electrochem. Commun., 2002, 4, 105-109.

56 A. Zolfaghari, F. Ataherian, M. Ghaemi and A. Gholami, Electrochim. Acta, 2007, 52, 2806-2814.

57 M. Ghaemi, F. Ataherian, A. Zolfaghari and S. M. Jafari, Electrochim. Acta, 2008, 53, 4607-4614.

58 V. Khomenko, E. Frackowiak and F. Béguin, Electrochim. Acta, 2005, 50, 2499-2506.

59 J. Wang, Y. Xu, X. Chen and X. Du, J. Power Sources, 2007, 163, 1120-1125.

60 H. An, Y. Wang, X. Wang, L. Zheng, X. Wang, L. Yi, L. Bai and X. Zhang, J. Power Sources, 2010, 195, 6964-6969.

61 Y. Fang, J. Liu, D. J. Yu, J. P. Wicksted, K. Kalkan, C. O. Topal, B. N. Flanders, J. Wu and J. Li, J. Power Sources, 2010, 195, 674-679.

62 S. H. Mujawar, S. B. Ambade, T. Battumur, R. B. Ambade and S.-H. Lee, Electrochim. Acta, 2011, 56, 4462-4466.

63 H. Zhang, G. Cao, Z. Wang, Y. Yang, Z. Shi and Z. Gu, Electrochem. Commun., 2008, 10, 1056-1059. 